C

овременные проблемы генно-инженерной биологической терапии больных псориазом

Бакулев А.Л.

Каседра дерматовенерологии и косметологии ФГБОУ ВО «Саратовский ГМУ им. В.И. Разумовского» Минздрава России 410028, г. Саратов, ул. Провиантская, д. 22

В статье приводится обзор литературы по актуальным проблемам применения генно-инженерных биологических препаратов (ГИБП) в лечении больных псориазом.

С современных позиций анализируются различные аспекты и проблемы применения ГИБП - цель терапии (PASI, BSA, контролируемая ремиссия, индивидуальная цель), возможности ее достижения с использованием ГИБП; понятия первичных и вторичных неответчиков на лечение ГИБП; вопросы иммуногенности и фракторы, влияющие на данный феномен; ускользание терапевтического ответа и его фрлюктуация в процессе долгосрочного применения ГИБП у больных псориазом.

Ключевые слова: псориаз, лечение, генно-инженерные биологические препараты, цель терапии, иммуногенность, эффективность, ускользание терапевтического ответа, флюктуация терапевтического ответа.

Конфрликт интересов: автор заявляет об отсутствии конфрликта интересов по данной публикации.

Для цитирования: Бакулев А.Л. Современные проблемы генно-инженерной биологической терапии больных псориазом. Вестник дерматологии и венерологии. 2020; 97 (2): 51—57. https://doi.org/10.25208/vdv1109 


\section{The Actual Challenges of Biologics Treatment in Patients with Psoriasis}

Andrey L. Bakulev

Department of Dermatovenereology and Cosmetology, Saratov State Medical University of the Ministry of Healthcare of the RF Proviantskaya str., bldg 22, Saratov, 410028, Russia

The article contain the main challenges meet doctors in real practice using biologics for treatment patients with psoriasis.

Different aspects and concerns have been analised from new point of view: the aims of Bx therapy (incl. control of remission, personalized goals, etc.) primary and secondary responses, immunogenicity, treatment survival, retention rate or long lasting regain of response in psoriasis patients treated by biologics.

Keywords: psoriasis, treatment, biologics products, treatment goal, immunogenicity, efficacy, lost of efficacy, retention rate of response.

Conflict of interest: the author state that there is no potential conflict of interest requiring disclosure in this article.

For citation: Andrey L. Bakulev. The Actual Challenges of Biologics Treatment in Patients with Psoriasis. Vestnik Dermatologii i Venerologii. 2020; 97 (2): 51—57. https://doi.org/10.25208/vdv1109 
Терапия среднетяжелых и тяжелых форм псориаза предполагает использование методов фототерапевтического воздействия (узкополосная средневолновая ультрафиолетовая терапия, фотохимиотерапия, бальнеофотохимиотерапия), системных лекарственных средств (метотрексат, циклоспорин, ароматические ретиноиды, апремиласт, генно-инженерные биологические препараты - ГИБП) [1-5].

Несмотря на высокую стоимость, ГИБП в последние годы стали использоваться в лечении больных псориазом значительно чаще, что связано с их таргетным действием на ключевые звенья патогенеза данного дерматоза, высокой терапевтической эффрективностью, приемлемой безопасностью, возможностью применения в долгосрочной перспективе, в том числе у лиц с резистентностью к другим методам и средствам лечения, способностью оказывать терапевтическое действие не только в отношении кожных проявлений заболевания, но также при ониходистрофиях и псориатическом артрите [5].

Существенно расширился перечень ГИБП для лечения псориаза у пациентов. В настоящее время применяются ингибиторы следующих интерлейкинов: TNF- $\alpha$ (инфликсимаб, адалимумаб, этанерцепт, цетролизумаб пэгол), IL-12/23 (устекинумаб), IL-23 (гуселькумаб) [5].

Одной из тенденций последних лет стало создание целой группы ГИБП, оказывающих селективное воздействие против IL-17. Так, в мире в течение нескольких последних лет применяются в условиях реальной клинической практики анти-IL-17 препараты секукинумаб, иксекизумаб, бродалумаб*, нетакимаб. Опубликованы данные рандомизированных клинических исследований по эффрективности и безопасности нового анTи-IL-17 лекарственного средства бимекизумаб* [6-7].

Расширение спектра ГИБП для лечения псориаза и увеличение потребности в их использовании из-за роста тяжелых фрорм дерматоза, резистентных к другим видам терапии, являются факторами, способствующими тому, что данный вид лечения перестает быть “уделом» узкого круга специалистов - дерматологов, уже имеющих опыт работы с ГИБП. В настоящее время появилась необходимость обсудить целый ряд практических вопросов, связанных с назначением/отменой терапии ГИБП, устойчивостью к ГИБП (выживаемостью), переключениями с одного вида лечения на другой.

Цель терапии. Псориаз относится к заболеваниям с неизвестной этиологией, в связи с чем говорить о возможности полного излечения от данной патологии в настоящее время не представляется возможным. С другой стороны, появление в последние годы таргетных препаратов для лечения пациентов, страдающих данным дерматозом, позволило добиться впечатляющих результатов как с точки зрения очищения кожного покрова от высыпаний во время обострений, так и для пролонгации ремиссии.

Следует понимать, что цели лечения с позиций пациентов и специалистов имеют существенные отличия. По данным M. Radke (2015), больные, страдающие псориазом, должны иметь уверенность в назначенной терапии, которая, в свою очередь, должна обеспечивать «свободу от высыпаний», контролировать течение заболевания, существенно улучшать качество их жизни и позволять «радоваться жизни». Специалисты дерматовенерологи фрормулируют свои ожидания от современных терапевтических средств для лечения псориаза иным образом: лекарственные препараты должны быть эфффективными, безопасными, удобными для применения, а также обеспечивать контроль течения не только клинических проявлений самого псориаза, но и коморбидных ему состояний (в первую очередь - кардиоваскулярной патологии) [8].

В настоящее время индекс PASI рассматривается как универсальный инструмент, с помощью которого специалисты оценивают эффективность предпринятого лечения у больных псориазом. На примере данного индекса можно проследить изменение возможностей лечения псориаза у пациентов от достижения клинически значимого ответа (PASI 50) в ответ на использование метотрексата, циклоспорина, ароматических ретиноидов и общей фотохимиотерапии с внутренним применением фотосенсибилизаторов до выраженного ответа (PASI 75), который стал возможен после появления в реальной клинической прак-

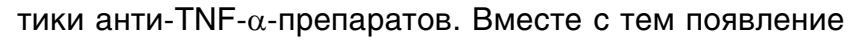
в арсенале практического здравоохранения анти-IL-17 и анти-IL-23 генно-инженерных биологических препаратов позволило добиваться более амбициозных целей лечения (PASI 90; PASI 100) [9].

Возможная цель терапии, которая в настоящее время обсуждается экспертами, это достижение значений индекса BSA 1\%. Вместе с тем данный индекс отражает лишь площадь поражения кожи, не учитывает локализацию и тяжесть эффрлоресценций и влияние дерматоза на качество жизни больных.

Контролируемая ремиссия - одна из возможных целей терапии псориаза у пациентов. Широко распространенная в ревматологии и гастроэнтерологии концепция «лечение до достижения цели» полностью соответствует данной стратегии, которая сводится к долгосрочному назначению высокоэффрективных и безопасных препаратов, позволяющих добиться клинической ремиссии псориаза и максимально ее пролонгировать. Важными достоинствами данного терапевтического подхода являются возможности обеспечения хорошего терапевтического результата, улучшение качества жизни пациентов, препятствование потери трудоспособности пациентами, а также контроль над течением коморбидных состояний. Опыт применения ГИБП при псориазе свидетельствует о том, что препараты ингибиторов группы IL-17 наиболее полно соответствуют данной стратегии. Однако опыт многолетнего применения данных лекарственных препаратов из группы ингибиторов фрактора некроза опухоли- $\alpha$ свидетельствует о доказанном в условиях реальной клинической практики возможном снижении терапевтического ответа при долгосрочном их применении [9, 10].

Более того, важным аспектом при выборе цели терапии является такой фактор, как стремление самого пациента к абсолютно чистой коже либо возможность иметь хорошее качество жизни при наличии определенных элементов сыпи на теле. В ряде случаев пациенты, как правило молодого возраста, не имеющие опыта лечения псориаза, желают любой ценой избавиться от всех его клинических проявлений на коже. В случае легких фрорм течения данного дерматоза без поражения «проблемных» участков кожного покрова, отсутствия псориатических ониходистрофий и артрита применение ГИБП нельзя считать оправданной стратегией лечения. Хотя, несомненно, индивидуализация цели терапии для каждого конкретного клинического случая 
в условиях реальной клинической практики проводится специалистами и сейчас.

Первичные неответчики на терапию. Оценка терапевтического ответа на применение ГИБП представляется чрезвычайно важной терапевтической задачей для специалистов дерматовенерологов на этапе инициирующей терапии. Именно по окончании периода инициации лечения ГИБП целесообразно выявить пациентов, не достигших PASI 50 (обычно в сроки 12-16 недель от начала курса ГИПБ). В этом случае рекомендуется произвести смену лекарственного препарата. При терапевтическом ответе по PASI $51-74 \%$ решение об изменении терапевтической стратегии либо ее дальнейшем продолжении принимается с учетом влияния проводимого лечения на качество жизни больного.

В настоящее время считается, что низкий ответ на инициирующую терапию является предиктором того, что применение ГИБП в долгосрочной перспективе будет малоэффективным, и вопрос об изменении терапевтического подхода все равно придется решать специалистам [11-12].

Вторичные неответчики на терапию. Иммуногенность. Снижение эфрфективности в процессе долгосрочного применения ГИБП именуют феноменом ускользания терапевтического ответа, а таких пациентов - вторичными неответчиками. В основе данного френомена лежат иммунологические процессы, связанные с образованием нейтрализующих антител к отдельным структурам молекулы ГИБП (Т-хелпер-зависимый гуморальный ответ). При этом так называемые не-нейтрализующие антитела связываются с эпитопами вне области связывания с лекарственным средством, поэтому они не влияют на терапевтическую эффрективность ГИБП.

Иммуногенность ГИБП определяется следующими фракторами:

а) структурой молекулы ГИБП;

б) стабильностью ГИБП и наличием в нем примесей;

в) индивидуальными особенностями пациента;

г) схемой применения, дозой и путем введения ГИБП, определяющих его концентрацию в сыворотке крови [13].

Частота образования антител, используемых ГИБП, может сильно различаться:

\begin{tabular}{ccc}
\hline Препарат & Тип антитела & $\begin{array}{c}\text { Частота образования антител } \\
\text { к лекарственному препарату, \% }\end{array}$ \\
\hline Адалимумаб & Человеческое & $0-51[14]$ \\
\hline Гуселькумаб & Человеческое & $5,5[15]$ \\
\hline Иксекизумаб & Гуманизированное & $9-17[16]$ \\
\hline Секукинумаб & Человеческое & $<1[14]$ \\
\hline Устекинумаб & Человеческое & $4-8,6[14]$ \\
\hline Этанерцепт & Человеческое & $2-5[14]$ \\
\hline
\end{tabular}

Наличие антител в организме не влияет на эффективность ГИБТ, пока они не снижают концентрацию лекарственного препарата ниже терапевтической. При длительном использовании ГИБП уровень терапевтического ответа может начать постепенно снижаться, что требует усиления терапии в виде комбинации ГИБП с метотрексатом. В настоящее время показано, что для ГИБП из группы ингибиторов TNF- $\alpha$ и IL-12/23 дополнительное назначение метотрексата с использованием стандартных доз и схем применения позволяет ослабить и (или) отсрочить возникновение периода клинически значимого снижения терапевтического ответа. Последнее связано как с прямым терапевтическим действием метотрексата на клинические проявления псориаза, так и с его способностью к торможению иммунологических процессов в организме, ответственных за выработку собственных аутоантител с нейтрализующей активностью к ГИБП [17].

Выживаемость терапии. Следует подчеркнуть, что современные анти-IL-17 ГИБП обладают благоприятным профилем по показателю иммуногенности и демонстрируют хорошие результаты по выживаемости как в рамках рандомизированных клинических исследований, так и из реальной клинической практики [14].

В частности, лекарственный препарат секукинумаб в рамках клинического исследования SCULPTURE обеспечивает 5-летнее сохранение терапевтического эфрфекта, оцененного по PASI 75/90/100, у пациентов с псориазом для индекса PASI 75 - в 85,5\% случаев, для индекса PASI 90 - в 66,4\%, для индекca PASI 100 - соответственно в 41\% наблюдений. При этом среднее улучшение среднего абсолютного PASI от первоначального в 1-й год наблюдения составило $91,1 \%$, а через 5 лет непрерывного применения препарата - 90,1\% [18].

Дополнительную информацию о факторах, влияющих на выживаемость терапии, можно получить при анализе регистров псориатических больных, которые ведутся в реальных условиях. Так, A. Egeberg и соавт. (2018) проанализировали информацию по данному показателю в рамках регистра DERMBIO (Дания) [19]. При анализе были обнаружены существенные различия в исходных характеристиках пациентов, получавших терапию секукинумабом или устекинумабом в рамках клинической практики. Несмотря на результаты, свидетельствующие о более хорошей выживаемости устекинумаба в долгосрочной перспективе при анализе всех случаев, включенных в регистр, было установлено, что устекинумаб использовался бионаивными пациентами в 541 (54\%) случаев, секукинумаб - лишь у 42 (21,5\%). В качестве ГИБП второй линии устекинумаб применяли 293 (29,2\%) больных, секукинумаб 43 (22,1\%). Устекинумаб был третьим препаратом переключения у 129 (12,9\%) лиц - участников регистра, секукинумаб - у 37 (19\%). Устекинумаб использовали в рамках четвертой линии терапии 23 (2,3\%) пациентов, секукинумаб - соответственно 35 (18\%). Наконец, устекинумаб выступал в качестве ГИБП пятой линии терапии лишь у 16 (1,6\%) пациентов, а секукинумаб у 38 (19,5\%). При этом среди участников регистра $47,7 \%$ пациентов в группе секукинумаба были переведены на лечение данным ГИБП с устекинумаба. При анализе сравниваемых когорт пациентов, получавших устекинумаб и секукинумаб, было установлено, что чем больше переключений ГИБП было в анамнезе у пациентов с псориазом, тем ниже оказывался терапевтический результат в долгосрочной перспективе. Таким образом, количество переключений с одного ГИБП на другой является важнейшим фрактором, влияющим на показатель выживаемости терапии.

Данная гипотеза нашла подтверждение в наблюдении израильских коллег [20]. Среди 1459 пациентов, получивших 3070 курсов биологической тера- 
пии, устекинумаб имел значительно более высокую общую выживаемость по сравнению с ингибиторами ФНО- $\alpha$ и секукинумабом. При этом кривые выживаемости терапии были схожими с данными регистра DERMBIO. Проведенный мультивариантный анализ данных, полученных в ходе многолетнего использования данного вида терапии, позволил выявить причины различий в выживаемости между устекинумабом и секукинумабом, которые определяются как линией лечения, так и ее доступностью, а не только биологическими характеристиками каждого лекарственного средства. Кроме этого, авторы наблюдали, что отрицательными предикторами выживаемости биологической терапии также были большой вес и женский пол. Несмотря на некоторые ограничения данного наблюдения, исследование пролило свет на важный фактор выживаемости терапии, который может повлиять также на клинические рекомендации. G Sholom et al. пришли к выводу, что на выживаемость биологических препаратов влияют в том числе факторы, связанные с особенностями системы здравоохранения, а не только свойствами биологических лекарственных средств. Данное наблюдение еще раз подтверждает важность следования клиническим рекомендациям. Так, использование секукинумаба в первую линию терапии в Германии, Великобритании [13, 14] и других странах обеспечивает высокие цифры эфффективности и выживаемости секукинумаба в терапии псориаза, подтвержденные локальными регистрами PROSPECT (Германия), BADBIR (Великобритания) и др. Согласно последним данным регистра BADBIR выживаемость терапии секукинумабом в реальной клинической практике составила $91 \%$ через 1 год и $82 \%$ через 2 года наблюдения соответственно, что полностью соответствует показателям выживаемости устекинумаба [15].

Таким образом, именно у бионаивных пациентов применение ГИБП позволяет добиться наиболее выраженного и длительного терапевтического ответа по показателю тяжести и распространенности псориаза на коже. В этой связи специалисты должны понимать, что решение о назначении терапии первого ГИБП должно быть обоснованным, тщательно взвешенным и предполагать возможность долгосрочного использования назначенного лекарственного средства [16].

Флюктуация (вариабельность) терапевтического ответа. Изменение выраженности ответа на терапию ГИБП не всегда является признаком его ускользания. M. Augustin и соавт. (2019) проанализировали варианты изменений терапевтического ответа у пациентов, принимавших участие в рандомизированных клинических исследованиях CLEAR и FIXTURE и получавших секукинумаб, устекинумаб или этанерцепт [24]. Авторы констатировали 4 вида терапевтического ответа на терапию ГИБП:

а) стойкий;

б) снижение эфрфективности с последующим его восстановлением;

в) снижение эффрективности без восстановления терапевтического ответа;

г) отсутствие эфффективности.

Авторы подчеркивают, что оценка изменений эфрфрективности предпринятой терапии ГИБП должна проводиться с учетом не менее трех визитов пациента с инъекциями лекарственного средства из группы ГИБП.
Было показано, что через 52 недели непрерывной терапии ГИБП в исследовании CLEAR 90,2\% пациентов с секукинумабом достигли стабильной эффективности без ее потери (в группе устекинумаба - 74,3\%). При этом $77,7 \%$ участников исследования демонстрировали стабильную эффективность на протяжении всего срока наблюдения без какого-либо снижения ответа (в группе устекинумаба - 59,9\% пациентов).

В рамках клинического исследования FIXTURE coответственно 83,5 и 66,4\% субъектов, получавших секукинумаб, имели стабильную эффективность без потери или снижения ответа (в группе этанерцепта - соответственно 58,3 и 42,6\% пациентов).

Снижение терапевтического ответа с его последующим восстановлением при продолжении лечения секукинумабом в рамках протокола CLEAR констатировали у 2,4\% пациентов (соответственно у 3,8\% лиц, получавших устекинумаб). В рамках исследования FIXTURE восстановление терапевтического ответа имело место у 2,8\% лиц, получавших секукинумаб (соответственно для группы этанерцепта данный показатель составил $6,1 \%)$. При этом такие пациенты не рассматривались как кандидаты для смены ГИБП или дополнительного назначения метотрексата.

Снижение терапевтического ответа без его последующего восстановления зарегистрировали у 2,4\% лиц, получавших секукинумаб, и у 10,3\% - устекинумаб (CLEAR). Данный показатель в протоколе FIXTURE coставил 7,7\% (для пациентов, получавших секукинумаб) и 12,6\% (для больных, получавших этанерцепт).

Наконец, терапевтический ответ отсутствовал в исследовании CLEAR в 5,1\% случаев (секукинумаб) и в $11,5 \%$ (устекинумаб), в исследовании FIXTURE в $6,1 \%$ (секукинумаб) и в 23\% (этанерцепт).

Таким образом, эфффективность секукинумаба и устекинумаба была стабильной в течение 52 недель лечения у большинства пациентов. Продолжение лечения данными ГИБП в ряде случаев приводило к восстановлению эффективности у ряда пациентов. Стойкая потеря терапевтического ответа при использовании секукинумаба и устекинумаба была редкой.

Данные из вышеназванной публикации представляются весьма важными для практических врачей, занимающихся лечением больных псориазом ГИБП. Оценка терапевтического ответа на ГИБП в долгосрочной перспективе обязательно должна включать анализ данных по эффективности как минимум за три визита пациента для инъекций лекарственного средства, что позволит минимизировать переключения и добиться лучших результатов терапии.

Эффрективность терапии VS безопасность. Одной из тенденций последних лет является тестирование больших доз ГИБП для достижения лучшего терапевтического ответа по показателям тяжести и распространенности псориатического процесса. В этом есть несомненная логика, так как более частое введение в организм больного псориазом массивного количества антител к тому или иному провоспалительному интерлейкину при связывании в терапевтическом плане позволяет добиться весьма амбициозных результатов. Однако данная проблема имеет и обратную сторону. Совсем недавно J. Bilal et al. (2018) опубликовали данные метаанализа эффрективности и безопасности ГИБП, в котором всесторонне проанализированы взаимосвязи дозировок ряда ГИБП с их 
эффрективностью в короткие сроки и безопасностью. Авторы метаанализа с удовлетворением констатируют высокие и схожие между собой показатели терапевтического ответа по индексам PGA и IGA 0/1 среди всех ингибиторов интерлейкинов (IL-12/23, IL-17 и селективных ингибиторов IL-23). Вместе с тем показано, что частота серьезных нежелательных явлений и случаев отмены лечения ГИБП не изменялась у лиц с псориазом, получавших устекинумаб, секукинумаб и бродалумаб. При этом высокий терапевтический ответ на иксекизумабе, который вводили чаще (один раз в две или четыре недели), сопровождался увеличением частоты отмены терапии из-за токсичности, что может быть связано как с особенностью молекулы и иммуногенностью иксекизумаба, так и с более высокой кратностью его введения в организм [25]. Результаты другого метаанализа Z.K. Jabbar-Lopez et al. (2017) также coгласуются с вышеприведенными данными: высокая эффрективность анти-IL-17 препаратов при псориазе по показателям достижения чистой/почти чистой кожи считается доказанной. Однако авторы также приходят к выводу о преимуществе секукинумаба перед иксекизумабом по профилю «польза/риск» [26].

Итак, применение ГИБП из экзотики становится реальным терапевтическим инструментом, позволяющим добиться высокой терапевтической эфрфективности при псориазе у больных, страдающих среднетяжелыми и тяжелыми формами данного заболевания.

Использование ГИБП позволяет добиться высокой терапевтической эфрфективности, соответствующей снижению абсолютных величин индекса PASI на 90$100 \%$ и пролонгировать такой терапевтический эффект в долгосрочной перспективе.

При первичном неответе на терапию ГИБП целесообразно рассмотреть вопрос о возможной смене ГИБП.

При долгосрочном лечении пациентов псориазом ГИБП возможно возникновение вторичного неответа на терапию и формирование френомена ускользания терапевтического ответа. Одним из факторов, препятствующим появлению аутоантител к ГИБП, считается назначение метотрексата.

Флюктуация терапевтического ответа на терапию с временным снижением эффективности и последующим восстановлением терапевтического ответа - феномен, не требующий смены ГИБП или дополнительного назначения метотрексата.

ГИБП секукинумаб является лекарственным препаратом, позволяющим добиться высокого терапевтического ответа у больных псориазом с удовлетворительным профилем безопасности. Возможная фрлюктуация (вариабельность) показателей тяжести и распространенности дерматоза в процессе долгосрочного лечения больных псориазом на примере применения секукинумаба является проявлением нормального терапевтического ответа. Для заключения об «ускользании» эффективности проводимой терапии ГИБП должно быть зафиксировано как минимум снижение PASI 50 в течение трех последующих визитов пациента, связанных с датами введения лекарственного средства в соответствии с инструкцией по его применению.

\section{Литература/References}

1. Elmets C.A., Lim H.W., Stoff B., Connor C., Cordoro K.M., Lebwohl M., Armstrong A.W., Davis DMR, Elewski B.E., Gelfand J.M., Gordon K.B., Gottlieb A.B., Kaplan D.H., Kavanaugh A., Kiselica M., Kivelevitch D., Korman N.J., Kroshinsky D., Leonardi C.L., Lichten J., Mehta N.N., Paller A.S., Parra S.L., Pathy A.L., Farley Prater E.A., Rupani R.N., Siegel M., Strober B.E., Wong E.B., Wu J.J., Hariharan V., Menter A. Joint American Academy of Dermatology-National Psoriasis Foundation guidelines of care for the management and treatment of psoriasis with phototherapy. J Am Acad Dermatol. 2019 Sep; 81 (3): 775—804.

2. Kogan N., Raimondo N., Gusis S.E., Izcovich A., Abarca Duran J.A., Barahona-Torres L., Blanco O., Quintana G.B., Briones M.C., Castro C., Castro Vargas E.G., Criniti J., Diez de Medina J.C., Franco M., Gómez M., Levrero V.P., Martínez López J.E., Valenzuela F. Latin American Clinical Practice Guidelines on the Systemic Treatment of Psoriasis SOLAPSO Sociedad Latinoamericana de Psoriasis (Latin American Psoriasis Society). Int J Dermatol. 2019 Aug; 58 Suppl 1: 4-28.

3. American Academy of Dermatology Work Group, Menter A., Korman N.J., Elmets C.A., Feldman S.R., Gelfand J.M., Gordon K.B., Gottlieb A., Koo J.Y., Lebwohl M., Leonardi C.L., Lim H.W., Van Voorhees A.S., Beutner K.R., Ryan C., Bhushan R. Guidelines of care for the management of psoriasis and psoriatic arthritis: section 6 . Guidelines of care for the treatment of psoriasis and psoriatic arthritis: case-based presentations and evidence-based conclusions. J Am Acad Dermatol. 2011 Jul; 65 (1): 137-74.

4. Nast A., Gisondi P., Ormerod A.D., Saiag P., Smith C., Spuls P.I., Arenberger P., Bachelez H., Barker J., Dauden E., de Jong E.M., Feist E., Jacobs A., Jobling R., Kemény L., Maccarone M., Mrowietz U., Papp K.A., Paul C., Reich K., Rosumeck S., Talme T., Thio H.B., van de Kerkhof P.,
Werner R.N., Yawalkar N. European S3-Guidelines on the systemic treatmen of psoriasis vulgaris--Update 2015--Short version-EDF in cooperation with EADV and IPC. J Eur Acad Dermatol Venereol. 2015 Dec; 29 (12): 2277_94.

5. Псориаз. Федеральные клинические рекомендации. Дерматовенерология 2015: Болезни кожи. Инсекции, передаваемые половым путем. 5-е изд., перераб. и доп. М.: Деловой экспресс, 2016: 415-470. [Psoriasis. National Clinical Guidelines. Dermatovenerology 2015: Skin disease. Sexually transmitted infections. 5 edition Delovoy Express, 2016; 415-470 (Russia).]

6. Natsis N.E., Gottlieb A.B. Bimekizumab for the treatment of psoriatic disease. Expert Opin Biol Ther. 2018 Dec; 18 (12): 1193-1197.

7. Papp K.A., Merola J.F., Gottlieb A.B., Griffiths CEM, Cross N., Peterson L., Cioffi C., Blauvelt A. Dual neutralization of both interleukin 17A and interleukin 17F with bimekizumab in patients with psoriasis: Results from BE ABLE 1, a 12-week randomized, double-blinded, placebo-controlled phase 2b trial. J Am Acad Dermatol. 2018 Aug; 79 (2): 277-286.

8. Radtke M.A., Reich K., Spehr C., Augustin M. Treatment goals in psoriasis routine care. Arch Dermatol Res. 2015 Jul; 307 (5): 445—9.

9. Nast A., Jacobs A., Rosumeck S., Werner R.N. Efficacy and Safety of Systemic Long-Term Treatments for Moderate-to-Severe Psoriasis: A Systematic Review and Meta-Analysis. J Invest Dermatol. 2015 Nov; 135 (11): 2641-2648.

10. Thaçi D., Blauvelt A., Reich K., Tsai T.F., Vanaclocha F., Kingo K., Ziv M., Pinter A., Hugot S., You R., Milutinovic M. Secukinumab is superior to ustekinumab in clearing skin of subjects with moderate to severe plaque psoriasis: CLEAR, a randomized controlled trial. J Am Acad Dermatol. 2015 Sep; 73 (3): 400—9. 
11. Strober B.E., Clay Cather J., Cohen D., Crowley J.J., Gordon K.B., Gottlieb A.B., Kavanaugh A.F., Korman N.J., Krueger G.G., Leonardi C.L. et al. A Delphi Consensus Approach to Challenging Case Scenarios in Moderate-to-Severe Psoriasis: Part 1. Dermatol Ther (Heidelb). 2012 Dec; 2 (1):1. Epub 2012 Mar 17.

12. Strober B.E., Clay Cather J., Cohen D., Crowley J.J., Gordon K.B., Gottlieb A.B., Kavanaugh A.F., Korman N.J., Krueger G.G., Leonardi C.L. et al A Delphi Consensus Approach to Challenging Case Scenarios in Moderate-to-Severe Psoriasis: Part. 2. Dermatol Ther (Heidelb). 2012 Dec; 2 (1): 2. Epub 2012 Mar 30.

13. Nast A. et al. Germany Guidelines S3-Leitlinie 013-001 „Therapie der Psoriasis vulgaris", 2017.

14. C. Smith et al. British Association of Dermatologists guidelines for biologic therapy for psoriasis 2017 doi: 10.1111/bjd.15665

15. Warren R.B. et al. J Invest Dermatol 2015; 135: 2632—40; Bewley et al. Poster PS-03 presented at 6th Annual Practical Symposium, 8-11 August 2019, Beawer Creek, Colorado.

16. Лила А.М., Мартынова Л.В. Генно-инженерные биологические препараты: проблема первичной и вторичной неэфффективности. Вестник Санкт-Петербургской медицинской академии последипломного образования. 2011; 3 (4): 153-60. [A. Lila, L. Martynova. Biologics: primary and secondary non responders. Vestnik of Sankt-Peterburg Medical Academy postgraduate education. 2011; 3 (4): 153—60 (Russia).]

17. Becher Strand et al/ Immunogenicity of biologics in chronic inflammatory diseases. A systematic review. BioDrugs 2017; 31 (4): 299_316.

18. Инструкция по медицинскому применению препарата Тремсея. Py ЛП-005686. [Sammary Product Characteristic of Tremfeya. RU LP005686 (Russia).]

19. Инструкция по медицинскому применению препарата Талс. РУ ЛП-005200. [Sammary Product Characteristic for Tals. RU LP-005200 (Russia).]

20. Бакулев А.Л. Стратегия «лечение до достижения цели» при псориазе. Актуальные вопросы устойчивости к биологической терапии. Вестник дерматологии и венерологии. 2016; (5): 32-38. [A. Bukulev.
Strategy of results-oriented treatment for patients with psoriasis. Relevant issues of biological therapy persistence. Vestnik dermatologii i venerologii. 2016; 5: 32—38 (Russia).]

21. Bissonnette R., Luger T., Thaçi D., Toth D., Lacombe A., Xia S., Mazur R., Patekar M., Charef P., Milutinovic M., Leonardi C., Mrowietz U. Secukinumab demonstrates high sustained efficacy and a favourable safety profile in patients with moderate-to-severe psoriasis through 5 years of treatment (SCULPTURE ExtensionStudy). J Eur Acad Dermatol Venereol. 2018 Sep; 32 (9): 1507-1514.

22. Egeberg A., Iversen L., Gniadecki R., Hvid L., Dam T.N., Bryld L.E., Skov L. Characteristics of patients receiving ustekinumab compared with secukinumab for treatment of moderate-to-severe plaque psoriasis - nationwide results from the DERMBIO registry. J Eur Acad Dermatol Venereol. 2017 Jul; 31 (7): 1183-1187.

23. Sholom G., Cohen A.D., Feldhamer I., Comaneshter D., Freud T., Pavlovsky L. Drug survival in patients with psoriasis is associated with the availability of biologics medications. JEADV 2020: Jan 17. doi: 10.1111/ jdv.16205. [Epub ahead of print]

24. Augustin M., Thaci D., Eyerich K., Pinter A., Radtke M., Lauffer F., Mrowietz U., Gerdes S., Pariser D., Lebwohl M., Sieder C., Melzer N., Reich K. Continued treatment with secukinumab is associated with high retention or regain of response. Br J Dermatol. 2019 Apr 10. doi: 10.1111/bjd.17991.

25. Bilal J., Berlinberg A., Bhattacharjee S., Trost J., Riaz I.B., Kurtzman DJB. A systematic review and meta-analysis of the efficacy and safety of the interleukin (IL)-12/23 and IL-17 inhibitors ustekinumab, secukinumab, ixekizumab, brodalumab, guselkumab and tildrakizumab for the treatment of moderate to severe plaque psoriasis. J Dermatolog Treat. 2018 Sep; 29 (6): $569-578$.

26. Jabbar-Lopez Z.K., Yiu ZZN, Ward V., Exton L.S., Mohd Mustapa M.F., Samarasekera E., Burden A.D., Murphy R., Owen C.M., Parslew R., Venning V., Warren R.B., Smith C.H. Quantitative Evaluation of Biologic Therapy Options for Psoriasis: A Systematic Review and Network Meta-Analysis. J Invest Dermatol. 2017 Aug; 137 (8): 1646-1654.

\section{Информация об авторе}

Бакулев Андрей Леонидович - д.м.Н., профрессор, заведующий кафедрой дерматовенерологии и косметологии ФГБОУ ВО «Саратовский ГМУ им. В.И. Разумовского» Минздрава России; e-mail: al_ba05@mail.ru

\section{Information about the authors}

Andrey L. Bakulev — Dr. Sci. (Medicine), Prof., Department of Dermatovenereology and Cosmetology, Saratov State Medical University of the Ministry of Healthcare of the RF; e-mail: al_ba05@mail.ru 